\title{
FACTOR SAVING INNOVATIONS AND FACTOR INCOME SHARES
}

\author{
Hernando Zuleta \\ Universidad de Rosario
}

April 11, 2007 


\begin{abstract}
We present an endogenous growth model where innovations are factor saving. Technologies can be changed paying a cost and technological change takes place only if the benefits are larger than the costs. Since the gains derived from factor saving innovations depend on factor abundance, biased innovations respond to changes in factors supply. Therefore, as an economy becomes more capital abundant agents try to use capital more intensively. Consequently, (a) the elasticity of output with respect to reproducible factors depends on the capital abundance of the economy and (b) the income share of reproducible factors increases as the economy grows. Another insight of the model is that in some economies the production function converges to an AK in the long run, while in others long-run growth is zero.

Journal of Economic Literature Classification: O11, O31, O33.
\end{abstract}

Keywords: endogenous growth, capital using and labor saving innovations, factor income shares. 


\section{Introduction.}

Economic historians provide evidence that during the Industrial Revolution there was capital-using and labor-saving technological change (Cain and Paterson (1981)). In the same vein, recent economic literature shows that during the last few decades, there has been human capital-using and raw labor-saving technological change (Krusell et. al. (2000) and Acemoglu (2002) among others). In addition, changes in factor abundance preceded technological changes in both historical moments and it seems that variations in factor abundance generated factor saving innovations.

Traditionally, technological change has been understood mainly as a change in Total Factor Productivity (TFP) (Romer (1986), Lucas (1988)). In empirical studies it is usually assumed that the Solow residual is explained by TFP (Barro and Sala-i-Martin (1992), among others). Here, we depart from this approach and present a model where TFP is constant but long-run growth can exist because of the endogeneity of the factor intensity ${ }^{1}$. We argue that technology is continuously evolving in order to take advantage from changes in factor abundance. We develop a growth model where the factor intensity of the technology used by the firms is determined by the factor abundance of the economy. In the same way, factor prices are determined by the marginal productivity of factors, therefore factor saving innovations affect factor income shares.

The main empirical evidence related to factor shares and the elasticity of

\footnotetext{
${ }^{1}$ Acemoglu (2002) and Hansen and Prescott (2002) propose models where the capital intensity of the technology is endogenous but none of them explains long run growth with the mechanism we propose.
} 
output with respect to factors are in line with our hypothesis:

1. In the field of empirical economic growth, Durlauf and Johnson (1995) find that as economies grow their technologies become more intensive in reproducible factors. This implies that the elasticity of output with respect to reproducible factors is higher in rich economies.

2. With regard to the behavior of factor income shares there are several observations to be pointed out:

i From 1870 to 1990 in the United States the share of land in Net National Product has been continuously reduced (see Rhee (1991) and Hansen and Prescott (2002)).

ii In USA, the share of raw labor in National Income was reduced from $10 \%$ to $5 \%$ over the past 50 years. Indeed, the US relative supply of skilled work has increased rapidly. However, the returns to college education have not fallen. On the contrary, there has been an increase in the college premium over this period (see Krueger (1999), Krusell et.al. (2000) and Acemoglu (2002) among others).

iii Labor income share does not decrease or increase with development (Gollin (2002)). However, the standard measure of labor income share includes raw labor and human capital income share. In the same way, the standard measure of capital income share includes land income share. Therefore, by analyzing the behavior of unskilled labor and land income share it seems that the income share of not 
reproducible factors (land and raw labor) decreased while the income share of reproducible factors increased during the last century.

iv Caselli and Feyrer (2006), separate reproducible physical capital from natural capital and calculate both the capital income share and the marginal productivity of capital. They find that the income share of produced physical capital is higher in rich countries.

To account for these facts we propose a model of biased technological change where factor income shares are endogenous. We use a standard set-up: CobbDouglas production function, homogeneous agents and infinite horizon. For simplicity, we consider only two factors, one reproducible (which includes physical capital, human capital, etc.) and one not reproducible (labor and land) and we call them capital and labor, respectively. For the purpose of the paper, the implications of the model remain the same regardless of these simplifying assumptions.

We analyze the consequences of allowing for factor using or factor saving technological change. In a market economy, capital using and labor saving technological change increases the optimal capital labor ratio, given factor prices. Thus, if we consider a Cobb-Douglas production function $\left(Y=A K^{\alpha} L^{1-\alpha}\right)$ an increase in $\alpha$ is capital using and labor saving.

Factor prices are determined by the marginal productivity of factors. As a consequence, labor saving innovations reduce the income share of workers and increase capital income share. In more general terms, the model predicts that the income share of not reproducible factors decreases with the stage of 
development, while the income share of reproducible factors grows with the stage of development.

We assume that technologies are embodied in capital goods and that different qualities of capital embody technologies with different capital intensities. Capital goods that embody more capital intensive technologies cost more. In this way, the cost of a change in the capital intensity of a technology can be understood as the cost of changing the quality of capital goods. As a result, capital abundant countries have more incentives to make labor-saving innovations. In the same way, countries where the capital intensity of the technology is higher have more incentives to save. This produces a virtuous circle driving the economy to long-run growth.

This paper differs from the existing literature in several ways. For example, Kennedy (1964), Zeira (1998), and Acemoglu (2002), among others, present models with endogenous biased and directed technological change. However, they use this concept to explain differences in productivity across countries, the behavior of wage dispersion and other related facts but they do not explain long run growth. Boldrin and Levine (2002) provide a model of perfect competition, where long run growth is completely explained by factor saving innovations but they don't consider the effect of technology on capital income share.

Zeira (2005) and Zuleta and Young (2006) provide two sector models where long run growth is completely explained by factor saving innovations. However, in both models the capital income share is relatively constant.

Finally, Peretto and Seater (2005) present a model where long run growth 
is explained by factor saving innovations. However, in their model markets are not competitive and savings rates are exogenous.

The paper at hand is also related to Bertola (1993), who explains how the share of reproducible factors affects positively the savings rate and, in this way, the economic growth. Here we model the behavior of factor shares in such a way that technological change affects capital income share and capital income share affects the incentives for technological change.

The rest of the paper is organized as follows. In the next section we explain in some detail the concept of capital-using and labor-saving technological change. In section 3 the model and its planner solution are presented. Finally, concluding comments are provided in section 4.

\section{Capital-using and Labor-saving Technological}

\section{Change}

A capital-using and labor-saving innovation is a change in the technological parameters such that, holding the factor prices constant, the optimal capital labor ratio is increased. For a Cobb-Douglas $\left(Y=A K^{\alpha} L^{1-\alpha}\right.$.) the optimal

capital labor ratio is: $k^{*}=\left(\frac{\alpha}{1-\alpha} \frac{w}{r}\right)$. Thus, increasing $\alpha$ is the only way to have capital-using and labor-saving technological change. According to Durlauf and Johnson (1995) $\alpha$ is higher in rich economies than in poor economies, so there is a reason to think that some capital-using and labor-saving technological change is taking place (recall that $k$ includes human capital). 


\subsection{Technological Change and elasticity of substitution between factors}

When we endogenize the capital intensity of the technology $(\alpha)$ using a CobbDouglas, the elasticity of substitution becomes a function of capital labor ratio and it is not constant. To see this, let us consider the technical rate of substitution (TRS) and derive the elasticity of substitution $(\varepsilon)$ :

The technical rate of substitution is given by, $T R S=\frac{\partial Y}{\partial L}\left(\frac{\partial Y}{\partial K}\right)^{-1}$ and the elasticity of substitution is given by, $\varepsilon=\frac{T R S}{k}\left(\frac{\partial T R S}{\partial k}\right)^{-1}$.

In the case of the Cobb Douglas production function: $T R S=\frac{\alpha}{1-\alpha} \frac{1}{k}$ so the elasticity is given by $\varepsilon=\frac{1}{1-\left(\frac{\partial\left(\frac{\alpha}{1-\alpha}\right)}{\partial k} \frac{1-\alpha}{\alpha}\right)}$.

Whenever the capital labor ratio affects positively the incentives for technological change, the elasticity of substitution is higher than one. In other words, if $\alpha$ is endogenous then the elasticity of substitution between factors is also endogenous.

\subsection{Old and new technologies}

If technologies with different capital intensities are available, depending on the initial capital abundance of the economy, it can be optimal to use more than one technology because, ceteris paribus, capital is more productive with the capitalintensive technology and labor is more productive with the labor-intensive technology.

Insert Figure 1 about here. 
Figure 1 illustrates this point. The lines $\alpha_{0}$ and $\alpha_{1}$ represent the combinations of capital and labor needed to produce one unit of output with technologies 0 and 1 respectively and the ray $k_{E}$ represents the capital labor ratio of the economy. Splitting the factors between the two technologies in such a way that the capital labor ratio in technology 0 is equal to $k_{0}$ and the capital labor ratio in technology 1 is equal to $k_{1}$ the economy can produce more than if it uses only one technology.

However, if there are many available technologies and if total factor productivity is the same for every technology then no more than two technologies are used. In particular, only the technologies with the highest and the lowest capital intensity are utilized. The intuition is that given a capital labor ratio higher than one, the most capital-intensive technology produces the maximum output per worker. Similarly, given a capital labor ratio lower than one, the most laborintensive technology produces the maximum output per worker. Therefore, only the most capital-intensive and the most labor-intensive technologies are used.

For simplicity we assume constant total factor productivity so that technical changes must be biased. Now, biased innovations are likely to respond to changes in factors supply, that is, as an economy becomes more capital abundant agents try to use capital in a more intensive fashion and, by the same token, in labor abundant economies agents try to use labor intensively.

Primitive economies are capital scarce and labor abundant and primitive technologies are likely to be labor intensive. Indeed, in economies where the main activities are hunting and gathering, raw labor income share is close to 
one. For this reason, in the rest of the paper we assume that the technology $A L$ $(\alpha=0)$ is always freely available. Thus, the production function can be written as $Y=A L_{1}+A K^{\alpha}\left(L-L_{1}\right)^{1-\alpha}$, where $L_{1}$ is the labor devoted to produce with technology one, $L-L_{1}$ the labor devoted to produce with technology CobbDouglas; and $\alpha$ is the capital share of the most capital-intensive technology.

\subsection{Capital, Production Function and Technology}

\subsubsection{The cost of changing technology}

We assume that there are different qualities of capital. Any type of capital embodies a technology $\alpha$ and capital goods that embody more capital intensive technologies are more costly. In particular, we assume that for $a$ units of output devoted to build capital goods of type $\alpha$, the number of capital goods is given by $K_{\alpha}=a+\ln (1-\alpha) \Phi$ where $\Phi$ is a measure for the size of the market. For simplicity we use population as a measure of the size of the market, so if $L_{i}$ is the amount of people consuming the good produced by firm $i$ then the output produced by a firm $i$ using $K_{\alpha}$ units of capital of type $\alpha$ is given by $A\left(a_{i}+\ln \left(1-\alpha_{i}\right) L_{i}\right)^{\alpha_{i}} l_{i}^{1-\alpha_{i}}$ where $l$ is labor devoted to produce with the capital intensive technology.

Note that the size of a capital-intensive firm $L_{i}$ can be different from the amount of labor in the firm $l_{i}$ because there are two technologies at work. Thus, the labor devoted to produce with the capital intensive technology is smaller than the population.

This function is arbitrary and it was chosen because of its tractability. How- 
ever the main results of the model do not depend on such an assumption. ${ }^{2}$

Now, the assumption that the cost of changing $\alpha$ depends positively on its value implies decreasing returns to scale in the production of technologies. This assumption may be justified in two ways. On the one hand, since the work of Charles Jones (1995) diminishing returns in innovation have been a standard assumption in growth models ${ }^{3}$. On the other hand, relaxing this assumption does not break the main predictions of the model. However, if there were no diminishing returns in innovation, rich economies would reach the $A K$ after a finite number of periods.

\subsubsection{Choosing technology}

Recall that only one capital intensive technology is used at a time. That is, only one type of capital is at work so we can drop the subindex $\alpha$ and write $K_{i}=a_{i}+\ln \left(1-\alpha_{i}\right) L_{i}$.

Markets are competitive so firms choose the technology in order to maximize output, $\max _{\alpha_{i}} A\left(a_{i}+\ln \left(1-\alpha_{i}\right) L_{i}\right)^{\alpha_{i}} l_{i}^{1-\alpha_{i}}$ s.t $\alpha_{i} \geq 0$. As a result, in the interior solution, the technology is given by, $\alpha_{i}=\frac{\frac{K_{i}}{L_{i}} \ln k_{i}}{1+\frac{K_{\alpha, i}}{L_{i}} \ln k_{i}}$ where $k_{i}$ is the capital labor ratio used by the firm $i$ to produce with the capital intensice technology $\alpha$.

Note that, holding the rest constant, any increase in the size of the firm affects $K_{i}$ and $L_{i}$ in the same proportions so, the equilibrium level of $\alpha$ is

\footnotetext{
${ }^{2}$ See Zeira (2005) or Peretto and Seater (2006) for different costs functions.

${ }^{3}$ Jones (1995) points out that while the number of scientists and engineers in the OECD economies rose in the last few decades, total factor productivity growth rates remained constant.
} 
independent of the size of the firm. If all firms use the same technology and face the same market prices then for any pair of firms $i$ and $j, k_{i}=k_{j}$ and $\frac{K_{i}}{L_{i}}=\frac{K_{i}}{L_{j}}=\frac{K}{L}$, where $\frac{K}{L}$ is capital per capita in the economy. Finally, we assume a population size of one, $L=1$, therefore, the equilibrium $\alpha$ (common to every firm) is

$$
\alpha=\max \left\{0, \frac{K \ln k}{1+K \ln k}\right\}
$$

Note that given the amount of assets $a$ and the units of labor $l$ there is only one $\alpha$ that satisfies equation 1 and, given that $K=a+\ln (1-\alpha)$, there is only one $K$ that satisfies equation 1 . Note also that, in the interior solution, $\alpha$ is an increasing function of $a$ and that $\alpha$ converges to one as $a$ goes to infinity,

$$
\begin{aligned}
\frac{\partial \alpha}{\partial a} & =\frac{(1-\alpha)^{2}(1+\ln (k))}{1+(1-\alpha)(1+\ln (k))}>0 \\
\lim _{a \rightarrow \infty} \alpha & =1
\end{aligned}
$$

\section{The Model.}

In this section we present the model and the main results. Given that markets are competive and there are no externalities, we concentrate on the planner solution.

Old technologies are available, therefore production and savings can be writ- 
ten as,

$$
Y_{t}=A\left(1-l_{t}\right)+A K_{t}^{\alpha_{t}} l_{t}^{1-\alpha_{t}} \quad \text { and } \quad s_{t}=A\left(1-l_{t}\right)+A K_{t}^{\alpha_{t}} l_{t}^{1-\alpha_{t}}-c_{t} .
$$

We assume $L=1$ so $s$ and $K$ shall be interpreted as savings and capital per capita.

Now, to model the choice of technology in continuous time, we define savings $s$ as the change in assets, $s_{t}=\dot{a}_{t}$, so $s_{t}=\dot{K}_{t}+\frac{\dot{\alpha}_{t}}{1-\alpha_{t}}$ and the changes in capital and technology are given by

$$
\begin{gathered}
\dot{K}_{t}=u_{t} s_{t} \\
\dot{\alpha}_{t}=\left(1-u_{t}\right)\left(1-\alpha_{t}\right) s_{t}
\end{gathered}
$$

where $u_{t}$ is the share of savings devoted to consumption and investment in reproducible factors of a given quality.

\subsection{The Command Optimum}

The planner problem is the standard one: maximize the present discounted utility of the representative agent. Savings, can be devoted to accumulate capital of a given quality or to change the technology. 


$$
\begin{aligned}
& \operatorname{Max} \int \log c_{t} e^{-\rho t} d t \\
\text { s.t. } \quad \dot{K}_{t}= & u_{t}\left(s_{t}\right) \\
\dot{\alpha}_{t} & =\left(1-u_{t}\right)\left(1-\alpha_{t}\right)\left(s_{t}\right) \\
l_{t},\left(1-l_{t}\right), K_{t} \geq & 0
\end{aligned}
$$

and the transversality condition

$$
\lim _{t \rightarrow \infty} \frac{e^{-\rho t}}{c_{t}} a_{t}=0
$$

To find the interior solution we combine the First Order Conditions. ${ }^{4}$ The optimal growth rate of consumption and the amount of labor devoted to produce with the capital-intensive technology are the following:

$$
\begin{aligned}
\frac{\dot{c}_{t}}{c_{t}} & =\alpha_{t} A\left(k_{t}\right)^{\alpha_{t}-1}-\rho \\
l_{t} & =K_{t}\left(1-\alpha_{t}\right)^{\frac{1}{\alpha_{t}}}
\end{aligned}
$$

Equation 8 cannot hold if the initial conditions are such that the capital stock of the economy is high compared with the population. If $K_{t}>\left(1-\alpha_{t}\right)^{-\frac{1}{\alpha_{t}}}$ then only the capital-intensive technology is used. In other words, $K_{t}<\left(1-\alpha_{t}\right)^{-\frac{1}{\alpha_{t}}}$ is a necessary condition to have interior solution. Note also that by combining

\footnotetext{
${ }^{4}$ The complete derivation of the model is presented in the Appendix 1.
} 
equations 8 and 4 we can express total output as

$$
Y_{t}=A\left(1+\alpha_{t}\left(1-\alpha_{t}\right)^{\frac{1-\alpha_{t}}{\alpha_{t}}} K_{t}\right) \text { so } \frac{\partial Y_{t}}{\partial K_{t}}=A \alpha_{t}\left(1-\alpha_{t}\right)^{\frac{1-\alpha_{t}}{\alpha_{t}}}
$$

Note that the production function is not concave in the amount of assets $a$. Therefore, in order to guarantee that the solution of the problem exists we need to assume that $A<2 \rho$ (the proof is presented in the Appendix 4). ${ }^{5}$

From the equations 1 and 8 we can get the relation between capital and technology:

$$
K_{t}=-\frac{\alpha_{t}}{\ln \left(1-\alpha_{t}\right)}\left(\frac{\alpha_{t}}{\left(1-\alpha_{t}\right)}\right)
$$

From equation 9 it follows that $\frac{\partial \alpha}{\partial K}>0$ and $\lim _{K \rightarrow \infty} \alpha=1$ so we can use equation 9 to plot the equilibrium relation between $K$ and $\alpha$ (see figure 2).

\section{Insert Figure 2 about here}

To save on notation from now on we call the right hand side of equation 9 $\bar{K}\left(\alpha_{t}\right)$.

The marginal productivity of a unit of product invested in capital must be equal to the marginal productivity of a unit of product invested in technology. We assume technology reversibility so equation 9 always holds.

Note that $K_{t}>\left(\frac{1}{1-\alpha_{t}}\right)^{\frac{1}{\alpha_{t}}}$ implies $K_{t}>\bar{K}\left(\alpha_{t}\right)$ (the proof is presented in the Appendix 2.) Therefore, when capital stock is so high that it is optimal to use only one technology, it is also optimal to reduce the stock of capital and

\footnotetext{
${ }^{5}$ In the Appendix 4 we also provide the conditions under which the problem is concave.
} 
increase the capital share. In other words, in the optimal path the economy uses two technologies $\left(l_{t}<1\right.$ for any $\left.t\right)$.

Now, combining equations 7 and 8 we can find the growth rate of consumption:

$$
\frac{\dot{c}_{t}}{c_{t}}=\alpha_{t} A\left(1-\alpha_{t}\right)^{\frac{1-\alpha_{t}}{\alpha_{t}}}-\rho
$$

Note that depending on the values of $\alpha_{t}, A$ and $\rho$ the growth rate of consumption can be positive or negative.

Let us define $\alpha_{m}$ as the technology such that the growth rate of consumption is equal to zero, namely,

$$
\alpha_{m}\left(1-\alpha_{m}\right)^{\frac{1-\alpha_{m}}{\alpha_{m}}}=\frac{\rho}{A}
$$

and $\bar{K}\left(\alpha_{m}\right)$ as the stock of capital such that when $\alpha=\alpha_{m}$ equation 9 holds with equality,

$$
\bar{K}\left(\alpha_{m}\right)=-\frac{\alpha_{m}^{2}}{\left(1-\alpha_{m}\right)} \frac{1}{\ln \left(1-\alpha_{m}\right)}
$$

Equations 11 and 12 indicate the levels of the state variables for which the discount rate is equal to the marginal productivity of savings. If $\alpha$ is high, then marginal productivity of capital is also high and there are incentives to accumulate capital. Similarly, if the stock of capital is high, then the marginal productivity of technology is also high and there are incentives to improve the technology.

If the initial conditions are such that $\alpha<\alpha_{m}$ then consumption growth rate 
is negative and it may be optimal to have negative savings. Note also that the growth rate of the economy, once $\alpha>\alpha_{m}$, increases as the economy grows. ${ }^{6}$

Coming back to the dynamics of the model, from equation 9 , in the interior solution the growth rate of $\alpha$ is a function of the growth rate of capital,

$$
\dot{\alpha}=\left(\frac{\partial K}{\partial \alpha}\right)^{-1} \dot{K}
$$

substituting $\dot{\alpha}_{t}, \dot{K}_{t}$ and $\alpha_{t}$ from equations 1, 2, and 9 we find the share of output devoted to consumption and physical capital accumulation.

$$
u_{t}=\frac{\left(1-\alpha_{t}\right)}{\left(\frac{\partial \alpha_{t}}{\partial K_{t}}+\left(1-\alpha_{t}\right)\right)} \quad \text { and } 1-u_{t}=\frac{\frac{\partial \alpha_{t}}{\partial K_{t}}}{\left(\frac{\partial \alpha_{t}}{\partial K_{t}}+\left(1-\alpha_{t}\right)\right)}
$$

Thus, $u_{t}$ is a function of capital and technology. Note that in the long-run $\frac{\partial \alpha_{t}}{\partial K_{t}}$ goes to zero, so $u_{t}$ converges to one (in the Appendix 1 we prove that $\lim _{\alpha \rightarrow 1} \frac{\partial \alpha_{t}}{\partial K_{t}} \frac{1}{\left(1-\alpha_{t}\right)}=0$ and $\left.\lim _{\alpha \rightarrow 0} \frac{\partial \alpha_{t}}{\partial K_{t}} \frac{1}{\left(1-\alpha_{t}\right)}=1\right)$.

\subsection{The long-run}

If the initial conditions are such that state variables are low $\left(\alpha<\alpha_{m}\right.$ and $\left.K<\bar{K}\left(\alpha_{m}\right)\right)$ consumption growth rate is negative. If the economy is poor it would need a lot of time and effort to get the technology $\alpha_{m}$. Therefore, for poor economies it can be optimal to consume the entire output. If $\alpha$ is high consumption growth rate is positive. As we show below, two candidates for optimal path may arise: one with a small amount of capital in the long-run

\footnotetext{
${ }^{6}$ This result, is consistent with the empirical evidence presented by Maddison (2003).
} 
and another with an infinite stock of capital. In the latter, $\alpha$ goes to one and capital, consumption and income per capita grow at the same rate, $A-\rho$.

In the following lines, we characterize the transition and study when one of the paths can be ignored. To do so we use the relation between state variables $\left(K_{t}=\bar{K}\left(\alpha_{t}\right)\right)$. For presentational purposes in this subsection we assume that equation 9 holds for every $t$.

We have expressed $u$ as a function of $K$, so it is possible to write the growth rate of consumption and the growth rate of $\alpha$ as functions of $\alpha$ and $c$. Using equations 2, 9 and 13, we find the growth rate of technology,

$$
\dot{\alpha}=\left(1-\alpha_{t}\right) \frac{s_{t}}{1+\left(1-\alpha_{t}\right) \frac{\partial K_{t}}{\partial \alpha_{t}}}
$$

From equation 14 it follows that: (i) If technology is constant $(\dot{\alpha}=0)$ then either savings are zero $(s=0)$ or the technology $A K$ is in use $(\alpha=1)$. (ii) If $\alpha<1$ and $s=0$ then $c_{t}=A+\alpha_{t} A\left(1-\alpha_{t}\right)^{\frac{1-\alpha_{t}}{\alpha_{t}}} \bar{K}\left(\alpha_{t}\right)$ so, when the growth rate of technology is equal to zero $c_{t}$ is an increasing function of $\alpha$.

In the same way, equation 10 expresses the growth rate of $c_{t}$ as a function of $\alpha_{t}$ and implies that consumption growth rate is zero when $\alpha=\alpha_{m}$, positive when $\alpha>\alpha_{m}$ and negative when $\alpha<\alpha_{m}$.

We can plot equations 14 and 10 (see Figure 3) and build a phase diagram which has on the axis capital-intensity, $\alpha$ and consumption, $c$.

Insert figure 3 about here

The figure is divided in four areas. In sectors 1 and 2 , consumption is 
higher than output $(s<0)$ so capital and decrease. In sector 1 consumption decreases while in sector 2 consumption grows. In sector 3 , the growth rate of consumption is negative and consumption is lower than output $(s>0)$ so, in the interior solution, capital and technology grow. In sector 4, the growth rate of consumption is positive and consumption is lower than output $(s>0)$ so, in the interior solution, capital and technology grow.

The first thing to note is that $\alpha=\alpha_{m}$ and $K=\bar{K}\left(\alpha_{m}\right)$ is a steady state. However, this steady state is not stable. Indeed, any small increase (decrease) in the level of assets would turn positive (negative) the consumption growth rate.

Note also that that $c=A, \alpha=K=0$ is a candidate for steady state because the marginal productivity of savings is lower than the discount rate so agents have incentives to have negative savings. However, there is no way to reduce the stock of capital.

Additionally, there exists another candidate for optimal path characterized by positive savings, capital accumulation, technological change and an infinite stock of capital in the long run. To determine which one is the optimal path we analyze initial conditions $\left(K_{0}\right.$ and $\left.\alpha_{0}\right)$, discount rate and TFP.

Recall that if $\alpha<\alpha_{m}$ and $K<\bar{K}\left(\alpha_{m}\right)$ then consumption growth rate is negative. In such circumstances, if total factor productivity is low, discount rate is higher than the marginal productivity of savings and the output is so low that the economy would need a lot of time saving before getting the technology $\alpha_{m}$. Therefore, it is better to consume entirely the output. In this case in steady there 
is no capital and output is fully consumed. This result is formally presented in proposition 1 .

Proposition 1 If $\rho \bar{K}\left(\alpha_{m}\right)>A$ and $K=0$ then the optimal level of labor in the capital-intensive sector is $l=0$ and the optimal level of consumption percapita is $c=A$.

The proof is in the Appendix 3.

\subsection{Path Dependence}

We already know that (i) If $\rho>A$ long-run growth is not possible. Indeed, $\alpha(1-\alpha)^{\frac{1-\alpha}{\alpha}}$ is increasing in $\alpha$ and it converges to one when $\alpha$ goes to one. Therefore, independently of the value of $\alpha$, the discount rate is always higher than the marginal productivity of capital. Thus if $\rho>A$ there is only one candidate for optimal path. (ii) If $\rho<A$ long-run growth is possible. However, depending on the initial conditions stagnation can be optimal.

In propositions 2 and 3 we identify sufficient conditions for stagnation and long-run growth to be optimal.

Proposition 2 For any $K_{0}<\bar{K}\left(\alpha_{m}\right)$ the economy converges optimally to a steady sate without capital.

The proof is in the Appendix 3.

The decision to save or consume depends on the discount rate $\rho$ and on the marginal productivity of savings ( $K$ or $\alpha$ ). If $\alpha$ and $K$ are small, the discount rate is higher than the marginal productivity of technology (and capital) and the 
output is so low that the economy would need a lot of time saving before getting the technology $\alpha_{m}$. Therefore, it is better to consume entirely the output.

Proposition 3 If $K_{0}>\bar{K}\left(\alpha_{m}\right)$ then the economy presents long-run growth.

The proof is straightforward. When $K>\bar{K}\left(\alpha_{m}\right)$ and $\alpha>\alpha_{m}$, the marginal productivity of savings is higher than the discount rate, therefore savings are used to increase $K$ and $\alpha$ and the consumption growth rate is positive.

Note that $K_{0}>\bar{K}\left(\alpha_{m}\right)$ implies $A>\rho$. Therefore, long run growth is not possible if $A<\rho$.

\section{Conclusions}

We present a model of economic growth where technological innovations are factor saving and endogenous and factor income shares are determined by technology.

We concentrate on the case of just one reproducible factor and one not reproducible factor. However, results remain the same regardless of such simplification. Assuming that technologies can be changed paying a cost, we find that capital abundant countries are more likely to increase capital intensity than poor economies. As a result, both the elasticity of output with respect to capital and the elasticity of substitution between factors depend on the relative factor abundance of the economy. We also show that capital abundance stimulates labor-saving innovations and that savings are higher in economies where the technology is more capital-intensive. These effects generate a virtuous circle 
driving the economy to long-run growth.

Secondly, we find that poor economies may converge to a steady state without reproducible factors.

Thirdly, since factor prices are given by marginal productivity, as economies grow, the income share of the reproducible factor grows while the income share of the not reproducible factor decreases. This prediction is consistent with the generally accepted result of constant labor income share. Indeed, human capital accumulation stimulates human capital-using innovations and increases human capital income share. The increase in human capital income share can counterweight the reduction in raw labor income share in such a way that total labor income share (including remuneration for human capital) remains constant. The same logic can be applied to land and physical capital.

Additionally, because of the behavior of the factor income shares, in economies where the technology is changing, the interest rate does not decrease as the capital labor ratio grows.

Acknowledgement 4 I am grateful to Veneta Andonova, Giuseppe Bertola, Michele Boldrin, Antonio Ciccone, Jose Vicente Rodríguez Mora, Tim Kehoe, Bernardo Kugler, Albert Marcet, Giovanni Pica and Xavier Sala-i-Martin for comments and suggestions. The usual caveat applies. 


\section{Appendix 1}

\section{The Command Optimum}

The planner maximizes the consumers utility subject to the resource constraints so the Hamiltonian is given by

$$
H=\log c_{t} e^{-\rho t}+\gamma u(s)+\mu(1-u)(1-\alpha)(s)+\phi_{L} l+\phi_{K} K
$$

The first order conditions of the problem are the following:

$$
\begin{aligned}
\frac{1}{c} e^{-\rho t}-u \gamma-(1-u)(1-\alpha) \mu & =0 \\
(\gamma-(1-\alpha) \mu)(s) & =0 \\
(u(\gamma)+(1-u)(1-\alpha) \mu) A\left((1-\alpha)(k)^{\alpha}-1\right)+\phi_{L} & =0 \\
\left(u+(1-u)(1-\alpha) \frac{\mu}{\gamma}\right) \alpha A(k)^{\alpha-1}+\frac{\phi_{K}}{\gamma} & =-\frac{\dot{\gamma}}{\gamma} \\
\left(u\left(\frac{\gamma}{\mu}\right)+(1-u)(1-\alpha)\right) A\left(K^{\alpha} L^{1-\alpha} \ln (k)\right)-(1-u)(s) & =-\frac{\dot{\mu}}{\mu}
\end{aligned}
$$

Assuming interior solution and combining the first order conditions we get,

$$
\begin{aligned}
\frac{\dot{c}}{c} & =\alpha A(k)^{\alpha-1}-\rho \\
k & =\left(\frac{1}{1-\alpha}\right)^{\frac{1}{\alpha}}
\end{aligned}
$$

Differentiating equation 16 we find $\frac{\dot{\gamma}}{\gamma}=-\frac{\dot{\alpha}}{(1-\alpha)}+\frac{\dot{\mu}}{\mu}$. Combining with equations $6,18,19$ and 21 ,

$$
K=\frac{\alpha}{\ln \left(\frac{1}{1-\alpha}\right)}\left(\frac{\alpha}{(1-\alpha)}\right)
$$


so $\frac{\partial K}{\partial \alpha}=2 \frac{\alpha}{\left(\ln \left(\frac{1}{1-\alpha}\right)\right)(1-\alpha)}-\frac{\alpha^{2}}{\left[\ln \left(\frac{1}{1-\alpha}\right)(1-\alpha)\right]^{2}}+\frac{\ln \left(\frac{1}{1-\alpha}\right)}{\ln \left(\frac{1}{1-\alpha}\right)(1-\alpha)^{2}}$. Combining with equation $22, \frac{\partial K}{\partial \alpha}=\frac{K}{1-\alpha}\left(\frac{2-\alpha}{\alpha}-\frac{1}{\ln \left(\frac{1}{1-\alpha}\right)}\right)$

Corner Solution $1 \phi_{L}>0(l=0)$

$(u(\gamma)+(1-u)(1-\alpha) \mu) A\left((1-\alpha)(k)^{\alpha}-1\right)+\phi_{L}=0$ so,

$(1-\alpha)(k)^{\alpha}<1$ and $K<l\left(\frac{1}{1-\alpha}\right)^{\frac{1}{\alpha}}$

Therefore, if $l=0$ then $K=0$

Corner Solution $2 \phi_{K}>0(K=0)$

Combining equations 15 and 16 we get

$\frac{1}{c} e^{-\rho t}-u \gamma-(1-u)(1-\alpha) \mu=0 \quad$ and $\quad(\gamma-(1-\alpha) \mu)(s)=\phi_{1-u}$

Therefore, if $s=0$ then $\phi_{1-u}=0$.

From 17, $A\left((1-\alpha)\left(K^{\alpha}-l^{\alpha}\right)=\frac{\phi_{L} l^{\alpha}}{u(\gamma)+(1-u)(1-\alpha) \mu}\right.$.

Therefore, if $K=0$ then $l=0$

\section{Behavior of $u$ and 1-u in the interior solution}

$u=\frac{(1-\alpha)}{\frac{\partial \alpha}{\partial K}+(1-\alpha)} \quad$ and $\quad 1-u=\frac{\frac{\partial \alpha}{\partial K}}{\frac{\partial \alpha}{\partial K}+(1-\alpha)}$

So $\quad u=\frac{\frac{\partial K}{\partial \alpha}(1-\alpha)}{1+\frac{\partial K}{\partial \alpha}(1-\alpha)} \quad$ and $\quad 1-u=\frac{1}{1+\frac{\partial K}{\partial \alpha}(1-\alpha)}$

and, $\frac{\partial u}{\partial \alpha}=\frac{\left(\frac{\partial^{2} K}{\partial \alpha^{2}}(1-\alpha)-\frac{\partial K}{\partial \alpha}\right)}{\left(1+\frac{\partial K}{\partial \alpha}(1-\alpha)\right)^{2}}>0$ and $\frac{\partial(1-u)}{\partial \alpha}=\frac{\frac{\partial K}{\partial \alpha}-\frac{\partial^{2} K}{\partial \alpha^{2}}(1-\alpha)}{\left(1+\frac{\partial K}{\partial \alpha}(1-\alpha)\right)^{2}}<0$

Share of savings devoted to capital accumulation

In this section we proof that $\lim _{\alpha \rightarrow 1} \frac{\partial \alpha}{\partial K} \frac{1}{(1-\alpha)}=0$ and $\lim _{\alpha \rightarrow 0} \frac{\partial \alpha}{\partial K} \frac{1}{(1-\alpha)}=1$.

The relation between capital $(K)$ and technology $(\alpha)$ in the interior solution is given by, $K=\frac{\alpha}{\ln \left(\frac{1}{1-\alpha}\right)}\left(\frac{\alpha}{(1-\alpha)}\right)$. Therefore, 


$$
\frac{\partial K}{\partial \alpha}=2 \frac{\alpha}{\left(\ln \left(\frac{1}{1-\alpha}\right)\right)(1-\alpha)}-\frac{\alpha^{2}}{\left[\ln \left(\frac{1}{1-\alpha}\right)(1-\alpha)\right]^{2}}+\frac{\alpha^{2}}{\ln \left(\frac{1}{1-\alpha}\right)(1-\alpha)^{2}} \text {. Combining with }
$$

equation 22 and rearranging,

$$
(1-\alpha) \frac{\partial K}{\partial \alpha}=K\left(\frac{2-\alpha}{\alpha}-\frac{1}{\ln \left(\frac{1}{1-\alpha}\right)}\right)
$$

1. Note that $\lim _{\alpha \rightarrow 1}\left(\frac{2-\alpha}{\alpha}-\frac{1}{\ln \left(\frac{1}{1-\alpha}\right)}\right)=1$. Therefore $\lim _{\alpha \rightarrow 1} \frac{\partial K}{\partial \alpha}(1-\alpha)=\infty$ and $\lim _{\alpha \rightarrow 1} \frac{\partial \alpha}{\partial K} \frac{1}{(1-\alpha)}=0$

2. Note that $\lim _{\alpha \rightarrow 0}\left(\frac{2-\alpha}{\alpha}-\frac{1}{\ln \left(\frac{1}{1-\alpha}\right)}\right)=\infty$ and $\lim _{\alpha \rightarrow 0} K=0$. Using L'Hopital theorem we find that $\lim _{\alpha \rightarrow 0} \frac{\alpha}{\ln \frac{1}{1-\alpha}}=1$, from where the following results are straight forward: (a) $\lim _{\alpha \rightarrow 0} 2 \frac{\alpha}{\ln \frac{1}{1-\alpha}}=2 \quad$ (b) $\lim _{\alpha \rightarrow 0}\left(\frac{\alpha}{\ln \frac{1}{1-\alpha}}\right)^{2} \frac{1}{1-\alpha}=1$ $\lim _{\alpha \rightarrow 0} \frac{\alpha}{\ln \frac{1}{1-\alpha}} \frac{\alpha}{1-\alpha}=0$. Therefore $\lim _{\alpha \rightarrow 0} \frac{\partial K}{\partial \alpha}(1-\alpha)=1$

\section{The behavior of $\frac{c}{K}$ along the optimal path}

Proposition 4: Along the optimal path $\frac{\dot{c}}{c}<\frac{\dot{K}}{K}$ for any value of the state variables and in the long run $\frac{c}{K}=\rho$.

\section{Proof.}

1. Suppose not, that is, $\frac{\dot{K}}{K}<\frac{\dot{c}}{c}$.

(i) If $\frac{\dot{K}}{K}<\frac{\dot{c}}{c}$ then the consumption-capital ratio $\frac{c}{K}$ grows with time

(ii) $\frac{\dot{c}}{c}-\frac{\dot{K}}{K}=\alpha A(1-\alpha)^{\frac{1-\alpha}{\alpha}}-\rho-\left(\frac{Y}{K}-\frac{c}{K}\right) u$, rearranging,

$\frac{\dot{c}}{c}-\frac{\dot{K}}{K}=\alpha A(1-\alpha)^{\frac{1-\alpha}{\alpha}}(1-u)+\left(\frac{c-A}{K}\right) u-\rho$

Therefore, $\frac{\dot{c}}{c}>\frac{\dot{K}}{K}$ iff $\frac{c}{K}>\frac{A}{K}+\frac{\rho}{u}-\alpha A(1-\alpha)^{\frac{1-\alpha}{\alpha}}\left(\frac{1-u}{u}\right)$

Note that $\frac{1}{u}=\frac{1-u}{u}+1$. Therefore,

$\frac{\dot{c}}{c}>\frac{\dot{K}}{K}$ iff $\frac{c}{K}>\frac{A}{K}+\rho+\left(\frac{1-u}{u}\right)\left[\rho-\alpha A(1-\alpha)^{\frac{1-\alpha}{\alpha}}\right]$

(iii) In the interior solution as $\mathrm{K}$ grows: 
- $\frac{A}{K}$ decreases

- $\frac{1-u}{u}$ decreases

- $\rho-\alpha A(1-\alpha)^{\frac{1-\alpha}{\alpha}}$ decreases

From (i), (ii) and (iii) it follows that if $\frac{c}{K}$ grows with $K$ then its growth rate becomes bigger.

Therefore, if $\frac{\dot{K}}{K}<\frac{\dot{c}}{c}$ then $\lim _{t \rightarrow \infty} \frac{c}{K}=\infty$ which is not feasible.

2. Since $\lim _{t \rightarrow \infty}\left(\frac{\dot{c}_{t}}{c_{t}}-\frac{\dot{K}_{t}}{K_{t}}\right)=\frac{c_{t}}{K_{t}}-\rho$ then in the long run $\frac{c}{K} \leq \rho$.

Now, if $\lim _{t \rightarrow \infty} \frac{c}{K}<\rho$ then $\lim _{t \rightarrow \infty}\left(\frac{\dot{c}_{t}}{c_{t}}-\frac{\dot{K}_{t}}{K_{t}}\right)<0$ so $\lim _{t \rightarrow \infty} \frac{c}{K}=0$ and $\lim _{t \rightarrow \infty} \frac{K}{c}=\infty$ which violates the transversality condition.

Therefore, $\lim _{t \rightarrow \infty} \frac{c}{K}=\rho$. 


\section{Appendix 2}

Use of technologies, $K>\left(\frac{1}{1-\alpha}\right)^{\frac{1}{\alpha}}$ implies $K>K(\alpha)$

$K(\alpha)=\frac{\alpha}{(1-\alpha)} \frac{1}{\ln \left(\frac{1}{1-\alpha}\right)}$. Therefore we have to prove that $\left(\frac{1}{1-\alpha}\right)^{\frac{1}{\alpha}}>\frac{\alpha}{(1-\alpha)} \frac{1}{\ln \left(\frac{1}{1-\alpha}\right)}$,

rearranging,

$\frac{1}{\alpha} \ln \left(\frac{1}{1-\alpha}\right)>(1-\alpha)^{\frac{1-\alpha}{\alpha}}$

(i) Define the function $g(\alpha)=\frac{1}{\alpha} \ln \left(\frac{1}{1-\alpha}\right)$. It is straightforward to see that:

$\lim _{\alpha \rightarrow 0} g(\alpha)=1 \quad$ and $\quad g^{\prime}(\alpha) \geq 0$

(ii) $(1-\alpha)^{\frac{1-\alpha}{\alpha}} \leq 1$

From (i) and (ii) it follows that $\frac{1}{\alpha} \ln \left(\frac{1}{1-\alpha}\right)>(1-\alpha)^{\frac{1-\alpha}{\alpha}}$. Therefore,

$$
\left(\frac{1}{1-\alpha}\right)^{\frac{1}{\alpha}}>\frac{\alpha}{(1-\alpha)} \frac{1}{\ln \left(\frac{1}{1-\alpha}\right)} .
$$




\section{Appendix 3}

\section{Proof of proposition 1:}

If $\rho \bar{K}\left(\alpha_{m}\right)>A$ given the initial level of capital $K=0$, the optimal levels of labor in the capital-intensive sector is $l=0$ and consumption percapita is $c=A$.

We already know that $K=0$ implies $l=0$ (see Appendix 1). Therefore it suffices to prove that $c=A$ is optimal.

Suppose $c \neq A$. We can ignore $c>A$ because it is not feasible.

1. If $c<A$ then $c<\rho \bar{K}\left(\alpha_{m}\right)$.

2. As long as $\alpha_{m} \geq \alpha_{t}$ the growth rate of consumption is equal or lower than zero.

3. From 1 and $2, c_{t}<\rho \bar{K}\left(\alpha_{m}\right)$ for any $t$ such that $\alpha_{m} \geq \alpha_{t}$.

Defining $T$ as the time needed to get the technology $\alpha_{m}$, namely, $\alpha_{T}=\alpha_{m}$, from 3 we know that $\frac{c_{T}}{K\left(\alpha_{T}\right)}<\rho$. But, in the interior solution the optimal consumption-capital ratio is higher than the discount rate, namely, $\frac{c_{t}}{K\left(\alpha_{t}\right)}>\rho$. for any $t$ (see proposition 4 ).

\section{Proof of proposition 2:}

For any $K_{0}<\bar{K}\left(\alpha_{m}\right)$ the economy converges optimally to a steady sate without capital.

Define $\tilde{\alpha}$ and $\tilde{K}$ as the levels of capital and technology such that output is equal to $\rho \bar{K}\left(\alpha_{m}\right)$, namely, $A\left(1+(1-\tilde{\alpha})^{\frac{1-\tilde{\alpha}}{\tilde{\alpha}}} \tilde{\alpha} \tilde{K}\right)=\rho \bar{K}\left(\alpha_{m}\right)$. 
Claim 1. If $K_{0}<\tilde{K}$ and $K_{0}<\bar{K}\left(\alpha_{m}\right)$ the economy converges optimally to a steady sate without capital.

Suppose not, that is, there exists a $K_{0}<\tilde{K}$ such that the economy presents long-run growth.

1. In order to have capital accumulation or technological change consumption must satisfy: $c_{0}<A\left(1+\left(1-\alpha_{0}\right)^{\frac{1-\alpha_{0}}{\alpha_{0}}} \alpha_{0} K_{0}\right)$.

2. In the interior solution, the consumption-capital ratio decreases as the stock of capital grows and converges to $\rho$ as capital goes to infinity. Moreover as long as $\alpha<\alpha_{m}$ and $K<\bar{K}\left(\alpha_{m}\right)$ the growth rate of consumption is negative. Therefore, for any $t$ such that $K_{t} \leq \bar{K}\left(\alpha_{m}\right)$ it must be true that $c_{t}<c_{0}$.

3. Since the consumption-capital ratio decreases with time and converges to $\rho$ in the long-run then in the optimal path $c_{t}>\rho K_{t}$ for any $t<\infty$.

From 2 and 3 it follows that given $K_{0}$, if there is an optimal path with long-run growth then $c_{0}>\rho \bar{K}\left(\alpha_{m}\right)$. From 1,2 and 3 it follows that output at period zero must be higher than $\rho \bar{K}\left(\alpha_{m}\right)$, namely, $A\left(1+\left(1-\alpha_{0}\right)^{\frac{1-\alpha_{0}}{\alpha_{0}}} \alpha_{0} K_{0}\right)>$ $\rho \bar{K}\left(\alpha_{m}\right)$, from where, $K_{0}>\tilde{K}$. Which contradicts the assumption $K_{0}<\tilde{K}$.

Claim 2: $\tilde{K} \geq \bar{K}\left(\alpha_{m}\right)$

Suppose not, that is $\tilde{K}<\bar{K}\left(\alpha_{m}\right)$ and $\tilde{\alpha}<\alpha_{m}$. Therefore, $\frac{\rho}{A(1-\tilde{\alpha})^{\frac{1-\tilde{\alpha}}{\tilde{\alpha}}}}>1$.

From the definition of $\tilde{K}$ it follows that $\tilde{K}=\frac{\frac{\rho}{A} \bar{K}\left(\alpha_{m}\right)-1}{(1-\tilde{\alpha})^{\frac{1-\tilde{\alpha}}{\tilde{\alpha}}}}$.

If $\frac{\rho}{A(1-\tilde{\alpha})^{\frac{1-\tilde{\alpha}}{\tilde{\alpha}} \tilde{\alpha}}}>1$ then $\frac{\frac{\rho}{A} \bar{K}\left(\alpha_{m}\right)-1}{(1-\tilde{\alpha})^{\frac{1-\tilde{\alpha}}{\tilde{\alpha}} \tilde{\alpha}}}>\bar{K}\left(\alpha_{m}\right)-\frac{1}{(1-\tilde{\alpha})^{\frac{1-\tilde{\alpha}}{\tilde{\alpha}} \tilde{\alpha}}}$ which implies $\tilde{K}>\bar{K}\left(\alpha_{m}\right)$ which contradicts the assumption $\tilde{K}<\bar{K}\left(\alpha_{m}\right)$.

From Claims 1 and 2 it follows that if $K_{0}<\bar{K}\left(\alpha_{m}\right)$ then the economy converges optimally to a steady sate without capital. 


\section{Appendix 4}

\section{Sufficient Conditions}

Case $1, u=1, \phi_{1-u}>0$.

$\alpha$ is constant and $K$ is the relevant state variable. Therefore the Hamiltonian can be written in the following way,

$$
H(t, c, K, \lambda)=\log (c) e^{-\rho t}+\lambda(Y-c)
$$

The logarithmic function is concave so, to verify Mangasarian conditions, it remains to prove that the function $g(K)=(Y-c)$ is concave in $K$.

$$
\frac{\partial g(K)}{\partial K}=\frac{\partial Y}{\partial K}
$$

and

$$
\frac{\partial^{2} g(K)}{\partial K^{2}}=\frac{\partial^{2} Y}{\partial K^{2}}=(\alpha-1) \alpha A k^{\alpha-2}<0
$$

Case $2,1<u<1$

We can use that $K=a+\ln (1-\alpha)$, rewrite the production function in the capital intensive sector as $Y=A(a+\ln (1-\alpha))^{\alpha} l^{1-\alpha}$ and get rid of the control variable $u$.

In the interior solution $\alpha$ is determined by the amount of assets $a$. Therefore, we reduce the problem to one state variable, $a$.

Define $c_{t}^{*}$ as the control variable that maximizes the Hamiltonian given the 
state variable $\left(a_{t}\right)$ and the shadow prices.

Now define the maximized Hamiltonian function:

$$
H^{0}\left(a_{t}, \lambda_{t}\right)=\log \left(c_{t}^{*}\right) e^{-\rho t}+\lambda_{t}\left(Y_{t}-c_{t}^{*}\right)
$$

The first derivative of the maximized Hamiltonian function is given by,

$$
\frac{\partial H^{0}}{\partial a}()=\frac{1}{c} \frac{\partial c_{t}^{*}}{\partial a} e^{-\rho t}+\lambda_{t}\left(\frac{\partial Y_{t}}{\partial a}-\frac{\partial c_{t}^{*}}{\partial a}\right)
$$

and the second derivative is given by,

$$
\frac{\partial^{2} H^{0}}{\partial a^{2}}()=\left(\frac{1}{c^{*}} \frac{\partial^{2} c^{*}}{\partial a^{2}}-\left(\frac{1}{c^{*}} \frac{\partial c^{*}}{\partial a}\right)^{2}\right) e^{-\rho t}+\lambda_{t}\left(\frac{\partial^{2} Y}{\partial a^{2}}-\frac{\partial^{2} c^{*}}{\partial a^{2}}\right)
$$

Given that in the interior solution $\lambda_{t}=\frac{1}{c} e^{-\rho t}$

$$
\frac{\partial^{2} H^{0}}{\partial a^{2}}()=-\left(\frac{1}{c^{*}}\right) e^{-\rho t}\left(\frac{1}{c^{*}}\left(\frac{\partial c^{*}}{\partial a}\right)^{2}-\frac{\partial^{2} Y}{\partial a^{2}}\right)
$$

Therefore if $\frac{1}{c^{*}}\left(\frac{\partial c^{*}}{\partial a}\right)^{2} \geq \frac{\partial^{2} Y}{\partial a^{2}}$ then the maximized Hamiltonian function is concave in $a$

Proposition 5: If $\frac{A}{2}<\rho<A$ then maximized Hamiltonian function is concave in a

Proof. We prove that $\frac{1}{c^{*}}\left(\frac{\partial c^{*}}{\partial a}\right)^{2} \geq \frac{\partial^{2} Y}{\partial a^{2}}$.

Claim 1: $\frac{\partial^{2} Y}{\partial a^{2}}=\frac{\partial Y}{\partial a} \frac{1}{K} \frac{(1-\alpha)(1+\ln (k))}{1+(1-\alpha)(1+\ln (k))}$

From equations 4 and 8 the production function can be written as, 


$$
\begin{aligned}
& Y=A\left(1+\alpha(1-\alpha)^{\frac{1-\alpha}{\alpha}} K\right) . \text { Replacing } K=a+\ln (1-\alpha) \text { we get } \\
& Y=A\left(1+\alpha(1-\alpha)^{\frac{1-\alpha}{\alpha}}(a+\ln (1-\alpha)) .\right. \text { Therefore, the derivative of the }
\end{aligned}
$$

production function with respect to $a$ is

$$
\frac{\partial Y}{\partial a}=A \alpha(1-\alpha)^{\frac{1-\alpha}{\alpha}}-A\left(\alpha(1-\alpha)^{\frac{1-2 \alpha}{\alpha}}+(1-\alpha)^{\frac{1-\alpha}{\alpha}} \ln (1-\alpha) \frac{K}{\alpha}\right) \frac{\partial \alpha}{\partial a} .
$$

Replacing $K$ from equation $9, \frac{\partial Y}{\partial a}=A \alpha(1-\alpha)^{\frac{1-\alpha}{\alpha}}$ and the second derivative is given by

$$
\frac{\partial^{2} Y}{\partial a^{2}}=-(1-\alpha)^{\frac{1-\alpha}{\alpha}} A \ln (1-\alpha) \frac{1}{\alpha} \frac{\partial \alpha}{\partial a}
$$

replacing $\frac{\partial \alpha}{\partial a}$ from equation 2

$$
\frac{\partial^{2} Y}{\partial a^{2}}=-(1-\alpha)^{\frac{1-\alpha}{\alpha}} A \ln (1-\alpha) \frac{1}{\alpha} \frac{(1-\alpha)^{2}(1+\ln (k))}{1+(1-\alpha)(1+\ln (k))}
$$

Recall that in the interior solution $K=-\frac{\alpha}{\ln (1-\alpha)}\left(\frac{\alpha}{(1-\alpha)}\right)$. Therefore, $\ln (1-\alpha)=$ $-\frac{\alpha}{K}\left(\frac{\alpha}{(1-\alpha)}\right)$ and

$$
\frac{\partial^{2} Y}{\partial a^{2}}=(1-\alpha)^{\frac{1-\alpha}{\alpha}} A \frac{1}{K} \frac{(1-\alpha)(1+\ln (k))}{1+(1-\alpha)(1+\ln (k))}
$$

so

$$
\frac{\partial^{2} Y}{\partial a^{2}}=\frac{\partial Y}{\partial a} \frac{1}{K} \frac{(1-\alpha)(1+\ln (k))}{1+(1-\alpha)(1+\ln (k))}
$$

Claim 2: $\frac{1}{c^{*}}\left(\frac{\partial c^{*}}{\partial a}\right)^{2}=\frac{1}{c^{*}}\left(\frac{\frac{\partial Y}{\partial a}-\rho}{\frac{\partial Y}{\partial a}+\frac{A}{K}-\frac{c}{k}} \frac{c}{k}\right)^{2}$

$\frac{1}{c^{*}}\left(\frac{\partial c^{*}}{\partial a}\right)^{2}=\frac{1}{c^{*}}\left(\frac{\dot{c}}{\dot{a}}\right)^{2}$ so $\frac{1}{c^{*}}\left(\frac{\partial c^{*}}{\partial a}\right)^{2}=\frac{1}{c^{*}}\left(\frac{\frac{\dot{c}}{\dot{c}}}{\frac{\dot{c}}{K}} \frac{c}{K}\right)^{2}$

Using equation 10 and the resources constraint $\frac{1}{c^{*}}\left(\frac{\partial c^{*}}{\partial a}\right)^{2}=\frac{1}{c^{*}}\left(\frac{\frac{\partial Y}{\partial a}-\rho}{\frac{\partial Y}{\partial a}+\frac{A}{K}-\frac{c}{K}} \frac{c}{K}\right)^{2}$.

Claim 3: If $A<2 \rho$ and $\alpha>\alpha_{\min }$ then $\frac{\frac{\partial Y}{\partial a}-\rho}{\frac{\partial Y}{\partial a}+\frac{A}{K}-\frac{c}{k}} \geq 1$ implies $\frac{\partial^{2} H^{0}}{\partial a^{2}}()<0$ 
From claims 1, 2 and 3 it follows that,

$$
\frac{\partial^{2} H^{0}}{\partial a^{2}}()=-\left(\frac{1}{c^{*}}\right)^{2} e^{-\rho t}\left(\left(\frac{\frac{\partial Y}{\partial a}-\rho}{\frac{\partial Y}{\partial a}+\frac{A}{K}-\frac{c}{K}} \frac{c}{K}\right)^{2}-\frac{\partial Y}{\partial a} \frac{c}{K} \frac{(1-\alpha)(1+\ln (k))}{1+(1-\alpha)(1+\ln (k))}\right)
$$

rearranging,

$$
\frac{\partial^{2} H^{0}}{\partial a^{2}}()=-\left(\frac{1}{c^{*}}\right)^{2} e^{-\rho t} \frac{c}{K}\left(\left(\frac{\frac{\partial Y}{\partial a}-\rho}{\frac{\partial Y}{\partial a}+\frac{A}{K}-\frac{c}{K}}\right)^{2} \frac{c}{K}-\frac{\partial Y}{\partial a} \frac{(1-\alpha)(1+\ln (k))}{1+(1-\alpha)(1+\ln (k))}\right)
$$

Therefore,

If $\left(\frac{\frac{\partial Y}{\partial a}-\rho}{\frac{\partial Y}{\partial a}+\frac{A}{K}-\frac{c}{k}}\right)^{2} \frac{c}{K}>\frac{\partial Y}{\partial a} \frac{(1-\alpha)(1+\ln (k))}{1+(1-\alpha)(1+\ln (k))}$ then $\frac{\partial^{2} H^{0}}{\partial a^{2}}()<0$

(i) Note that $\frac{(1-\alpha)(1+\ln (k))}{1+(1-\alpha)(1+\ln (k))}=\frac{(1-\alpha)\left(1+\frac{\ln \left(\frac{1}{1-\alpha}\right)}{\alpha}\right)}{1+(1-\alpha)\left(1+\frac{\ln \left(\frac{1}{1-\alpha}\right)}{\alpha}\right)}<\frac{2}{3}$. Moreover, if $\alpha>\alpha_{\min }$ and $A<2 \rho$ then $\alpha>0,772$ so $\frac{(1-\alpha)(1+\ln (k))}{1+(1-\alpha)(1+\ln (k))}<\frac{2}{5}$. Therefore, $\frac{\partial Y}{\partial a} \frac{(1-\alpha)(1+\ln (k))}{1+(1-\alpha)(1+\ln (k))}<\frac{2}{5} \frac{\partial Y}{\partial a}$.

(ii) $\frac{\partial Y}{\partial a} \leq A$ and $A<2 \rho$ so $\frac{\partial Y}{\partial a} \leq 2 \rho$.

(iii) From (i) and (ii) it follows that $\frac{\partial Y}{\partial a} \frac{(1-\alpha)(1+\ln (k))}{1+(1-\alpha)(1+\ln (k))}<\frac{4}{5} \rho$. Therefore, given that $\frac{c}{K} \geq \rho$

If $\frac{\frac{\partial Y}{\partial a}-\rho}{\frac{\partial Y}{\partial a}+\frac{A}{K}-\frac{c}{k}} \geq 1$ then $\left(\frac{\frac{\partial Y}{\partial a}-\rho}{\frac{\partial Y}{\partial a}+\frac{A}{K}-\frac{c}{K}}\right)^{2} \frac{c}{K}>\frac{\partial Y}{\partial a} \frac{(1-\alpha)(1+\ln (k))}{1+(1-\alpha)(1+\ln (k))}$ and $\frac{\partial^{2} H^{0}}{\partial a^{2}}()<0$

Claim 4: $\frac{\frac{\partial Y}{\partial a}-\rho}{\frac{\partial Y}{\partial a}+\frac{A}{K}-\frac{c}{k}} \geq 1$.

(i) In the long run $\frac{A}{K}$ goes to zero and $\frac{c}{K}$ goes to $\rho$ so $\lim _{a \rightarrow \infty}\left(\frac{\frac{\partial Y}{\partial a}-\rho}{\frac{\partial Y}{\partial a}+\frac{A}{K}-\frac{c}{K}}\right)=1$. 
(ii) If $\frac{c}{k}-\frac{A}{K} \geq \rho$ then $\left(\frac{\frac{\partial Y}{\partial a}-\rho}{\frac{\partial Y}{\partial a}+\frac{A}{K}-\frac{c}{K}}\right) \geq 1$.

(iii) Along the transition the variables $c$ and $K$ grow. Therefore $\frac{A-c}{K}$ decreases so if $\frac{c_{t}}{K_{t}}-\frac{A}{K_{t}} \geq \rho$ then $\frac{c_{t-j}}{K_{t-j}}-\frac{A}{K_{t-j}} \geq \rho$ for any $j>0$.

From claims 1 to 4 it follows that if $\frac{A}{2}<\rho<A$ then $\frac{1}{c^{*}}\left(\frac{\partial c^{*}}{\partial a}\right)^{2} \geq \frac{\partial^{2} Y}{\partial a^{2}}$ and the maximized Hamiltonian function is concave in $a$

Proposition 6: If $A<2 \rho$ then the maximized Hamiltonian has a finite-value solution.

Proof. The maximized Hamiltonian is given by $H^{0}\left(a_{t}, \lambda_{t}\right)=u\left(c_{t}^{*}\right) e^{-\rho t}+$ $\lambda_{t}\left(Y_{t}-c_{t}^{*}\right)$. Therefore, we have to prove that $\lim _{t \rightarrow \infty} u\left(c_{t}^{*}\right) e^{-\rho t}=0$ and $\lim _{t \rightarrow \infty}$ $\lambda_{t}\left(Y_{t}-c_{t}^{*}\right)=0$. To simplify notation we drop the index *.

Claim 1: $\lim _{t \rightarrow \infty} u\left(c_{t}\right) e^{-\rho t}=0$

Note that $\lim _{t \rightarrow \infty} u\left(c_{t}\right)=\infty$ and $\lim _{t \rightarrow \infty} e^{-\rho t}=0$ so in order to find the limit we differentiate the expression $u\left(c_{t}\right) e^{-\rho t}: \frac{U^{\prime}\left(c_{t}\right)}{U\left(c_{t}\right)} \dot{c}_{t}-\rho$

Recall that the log utility function is a special case of the more general function CRRA, $\frac{c^{1-\sigma}}{1-\sigma}$. Indeed, $\lim _{\sigma \rightarrow 1} \frac{c^{1-\sigma}}{1-\sigma}=\log c^{7}$, so $\frac{U^{\prime}\left(c_{t}\right)}{U\left(c_{t}\right)} \dot{c}_{t}-\rho=\frac{1}{\sigma} \frac{\dot{c}_{t}}{c_{t}}-\rho$

Now, we use the log utility function so $\sigma=1$ and $\frac{U^{\prime}\left(c_{t}\right)}{U\left(c_{t}\right)} \dot{c}_{t}-\rho=\frac{\dot{c}_{t}}{c_{t}}-\rho$.

\footnotetext{
${ }^{7}$ To show that the utility function converges to logarithmic as $\sigma \rightarrow 1$ we make use of L'Hospital's rule. As $\sigma \rightarrow 1$, the numerator and denominator of the function both approach zero. Differentiate both the numerator and the denominator with respect to $\sigma$ and then take the limit of the derivatives' ratio as $\sigma \rightarrow 1$.
} 
From equation $10 \frac{\dot{c}_{t}}{c_{t}}=\alpha_{t} A\left(1-\alpha_{t}\right)^{\frac{1-\alpha_{t}}{\alpha_{t}}}-\rho$ so

$$
\begin{aligned}
\frac{U^{\prime}\left(c_{t}\right)}{U\left(c_{t}\right)} \dot{c}_{t}-\rho & =\alpha_{t} A\left(1-\alpha_{t}\right)^{\frac{1-\alpha_{t}}{\alpha_{t}}}-2 \rho, \\
\lim _{t \rightarrow \infty}\left(\frac{U^{\prime}\left(c_{t}\right)}{U\left(c_{t}\right)} \dot{c}_{t}-\rho\right) & =A-2 \rho \text { and } \\
\frac{U^{\prime}\left(c_{t}\right)}{U\left(c_{t}\right)} \dot{c}_{t}-\rho & \leq A-2 \rho \text { for every } t
\end{aligned}
$$

Therefore, if $A<2 \rho$ then $\lim _{t \rightarrow \infty}\left(\frac{U^{\prime}\left(c_{t}\right)}{U\left(c_{t}\right)} \dot{c}_{t}-\rho\right)<0$ and $\lim _{t \rightarrow \infty} u\left(c_{t}\right) e^{-\rho t}=0$.

Claim 2: $\lim _{t \rightarrow \infty} \lambda_{t}\left(Y_{t}-c_{t}\right)=0$.

1. From equation 10 in the appendix $\lambda_{t}\left(Y_{t}-c_{t}\right)=e^{-\rho t}\left(\frac{Y_{t}}{c_{t}}-1\right)$.

2. From proposition $5 \lim _{t \rightarrow \infty} \frac{c}{Y}=\frac{\rho}{A}$, so $\lim _{t \rightarrow \infty}\left(Y_{t}-c_{t}\right)=\left(\frac{A}{\rho}-1\right)$

3. $\lim _{t \rightarrow \infty} e^{-\rho t}=0$

From 1, 2 and 3 it follows that $\lim _{t \rightarrow \infty} \lambda_{t}\left(Y_{t}-c_{t}\right)=0$ 


\section{References}

[1] Acemoglu, Daron "Directed Technical Change" The Review of Economic Studies, 2002, 69 (4), 781-809.

[2] Barro, Robert and Sala-i-Martin, Xavier "Convergence" Journal of Political Economy, 1992, 100 (2), 223-251.

[3] Bertola, Giuseppe. "Factor Shares and Savings in Endogenous Growth" The American Economic Review,1993, 83 (5), 1184-1198.

[4] Boldrin, Michele and Levine, David K. " Factor Saving Innovation" Journal of Economic Theory, 2002, 105 (1), 18-41

[5] Boldrin, Michele and Levine, David K. "Perfectly Competitive Innovation," CEPR, 2002a, 3274, C.E.P.R. Discussion Papers.

[6] Cain, Louis P. and Paterson Donald G. "Factor Biases and Technical Change in Manufacturing: The American System, 1850-1919" The Journal of Economic History, 1981, 41, (2), 341-360

[7] Caselli, Francesco and Feyrer, James "The Marginal Product of Capital" Quarterly Journal of Economics, 2007, forthcoming.

[8] Durlauf, Steven and Johnson, Paul "Multiple Regimes and Cross-Country Growth Behavior" Journal of Applied Econometrics, 1995, 10 (4), 365-384.

[9] Gollin, Douglas "Getting Income Shares Right" Journal of Political Economy, 2002, $110(2), 458-474$. 
[10] Hansen, Gary and Prescott, Edward., "From Malthus to Solow", The American Economic Review, 2002, 92 (4), 1205-1217.

[11] Kennedy, Charles "Induced Bias in Innovation and the Theory of Distribution", Economic Journal, 1964, LXXIV, 541-547.

[12] Krueger, Alan "Measuring Labor's Share" The American Economic Review, 1999, 89 (2), 45-51.

[13] Krusell, Per; Ohanian, Lee; Ríos-Rull, José Victor and Violante, Giovani "Capital-Skill Complementarity and Inequality: A Macroeconomic Analysis", Econometrica, 2000, 68 (5) 1029-53.

[14] Lucas, Robert, "On the Mechanics of Economic Development", Journal of Monetary Economics, 1988, 22 (1), 3-41.

[15] Maddison, A., 2003. The World Economy: Historical Statistics, OECD Development Center.

[16] Peretto, Pietro and Seater, John "Augmentation or Elimination?", working paper 2006, http://ideas.repec.org/p/deg/conpap/c011_060.html

[17] Rhee, Changyong "Dynamic Inefficiency in an Economy with Land" Review of Economic Studies, 1991, 58 (4) 791-797.

[18] Romer Paul, "Increasing Returns and Long-run Growth", Journal of Political Economy, 1986, 94 (5), 102-137.

[19] Zeira, Joseph, "Workers, Machines and Economic Growth" Quarterly Journal of Economics, 1998, 113 (4), 1091-1118. 
[20] Zeira, Joseph, "Machines as Engines of Growth," CEPR 2005, Discussion Papers 5429. 


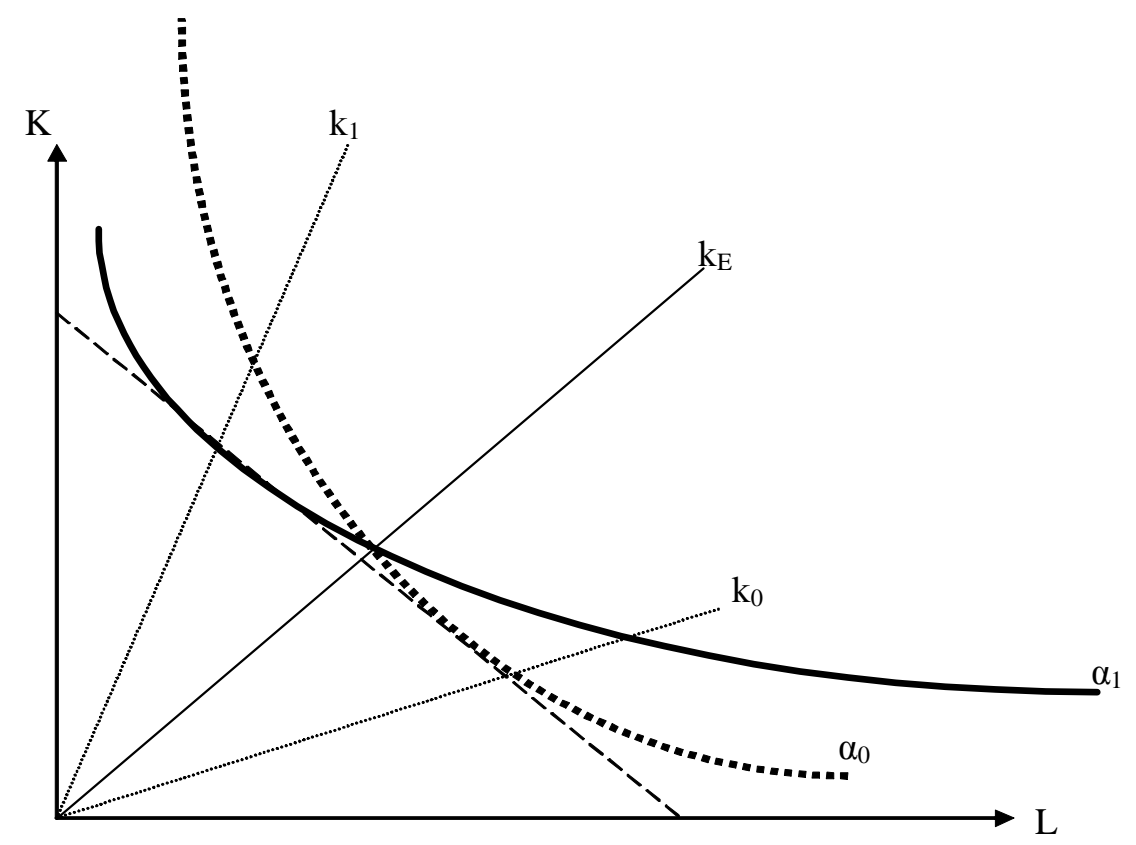




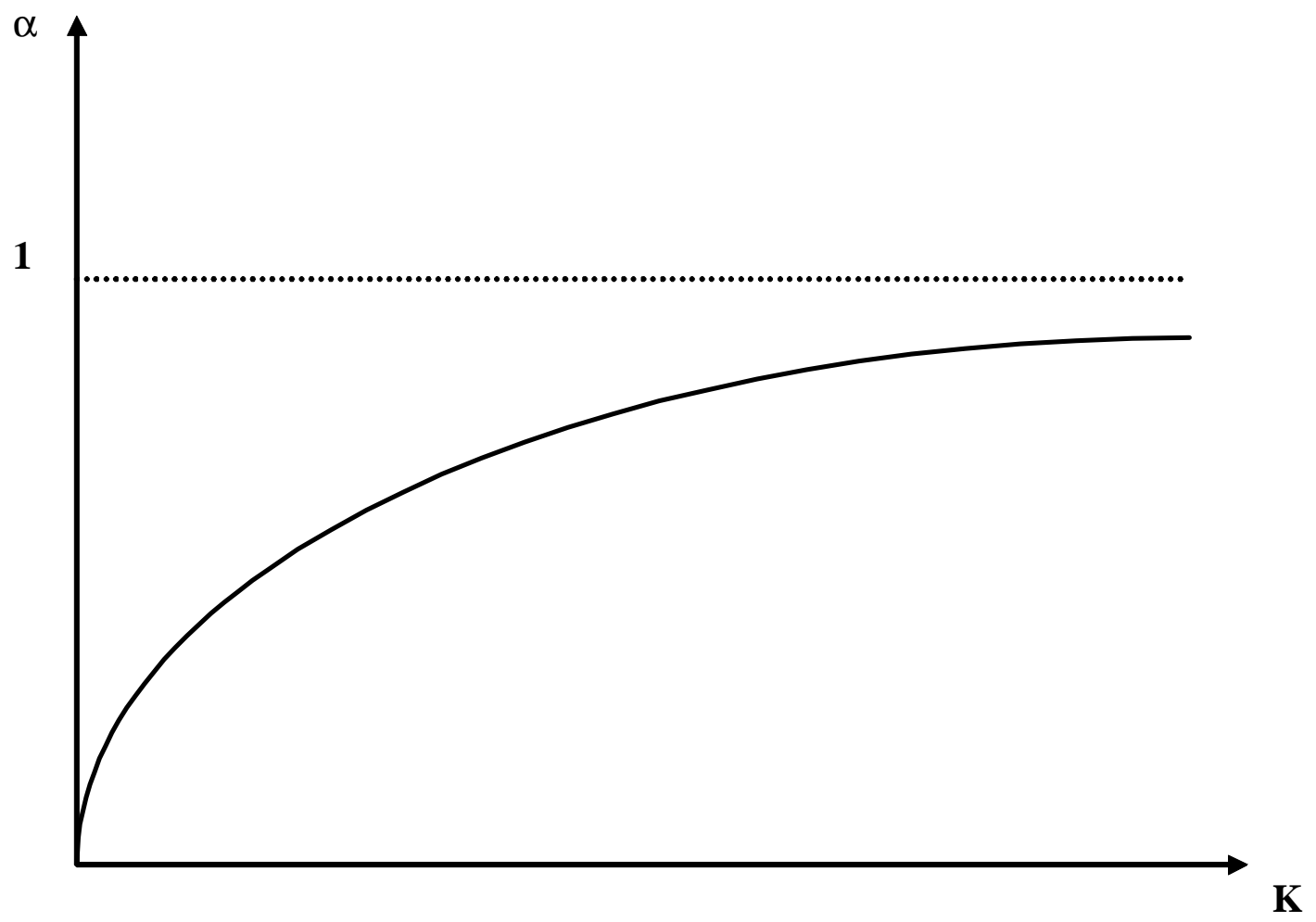

38 


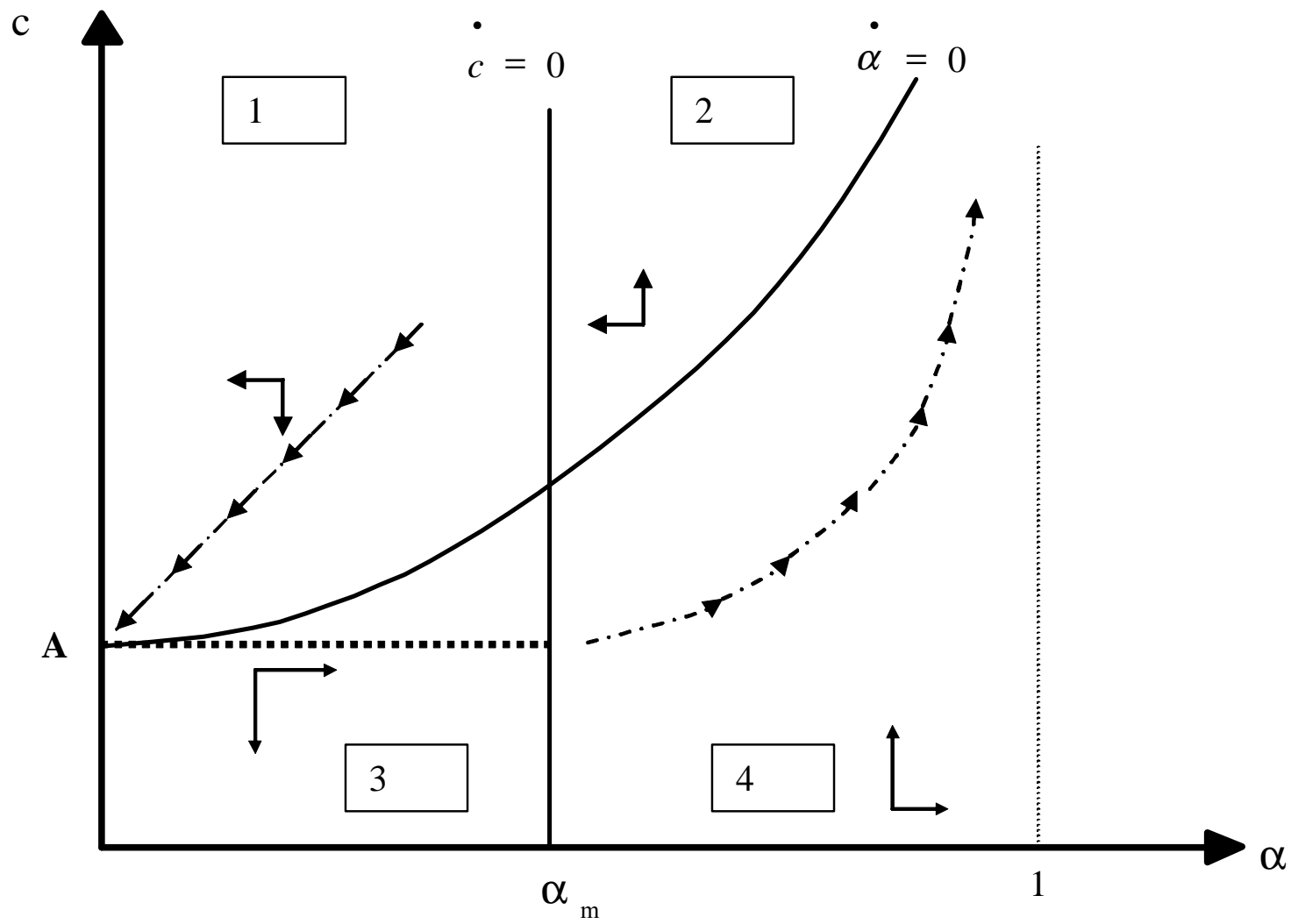

